\title{
Theta Dependence in Yang-Mills from Holography
}

\author{
Francesco Bigazzi ${ }^{1, \star}$, Aldo L. Cotrone ${ }^{1,2}$, and Roberto Sisca ${ }^{3}$ \\ ${ }^{1}$ INFN, Sezione di Firenze; Via G. Sansone 1, I-50019 Sesto Fiorentino (Firenze), Italy \\ ${ }^{2}$ Dipartimento di Fisica e Astronomia, Università di Firenze; Via G. Sansone 1, I-50019 Sesto Fiorentino \\ (Firenze), Italy \\ ${ }^{3}$ Department of Mathematics, University of Surrey; Guildford, GU2 7XH, UK
}

\begin{abstract}
In this contribution we review how holographic tools can be used to study the dependence on the CP-breaking $\theta$ parameter of various observables in a large $N_{c}$ Yang-Mills model. Relevant examples are the ground-state energy density, the string tension, the glueball mass spectrum and the critical temperature for deconfinement. These observables have been also studied, in the small $\theta$ regime and up to very few powers of $\theta^{2}$, in pure Yang-Mills on the Lattice. The holographic results qualitatively agree with available Lattice data and, being exact in $\theta$, provide benchmarks for higher order corrections.
\end{abstract}

\section{Introduction}

Instanton configurations naturally induce a topological term in the Lagrangian for a $S U\left(N_{c}\right)$ YangMills gauge theory

$$
\mathcal{L}_{E}=\frac{N_{c}}{2 \lambda}\left[\operatorname{Tr} F_{\mu \nu} F^{\mu \nu}-i \frac{\lambda}{16 \pi^{2}} \frac{\theta}{N_{c}} \epsilon^{\mu \nu \rho \sigma} \operatorname{Tr} F_{\mu \nu} F_{\rho \sigma}\right] .
$$

Here $\lambda=g^{2} N_{c}$ is the 't Hooft coupling and Euclidean signature is used. The $\theta$ parameter multiplies the so called topological charge density, a term which breaks parity and time reversal (hence $\mathrm{CP}$ ) symmetries. Since the $4 \mathrm{~d}$ integral of the topological charge density is an integer, the $\theta$ parameter behaves like an angle: the physics has to be periodic under $\theta \rightarrow \theta+2 \pi$ shifts.

Studying how observables depend on $\theta$ is both interesting and challenging. It is interesting because the parameter affects the vacuum structure of the theory. It is challenging because of the nonperturbative nature of the $\theta$ term. The use of numerical simulations on Euclidean Lattices is limited since the $\theta$ term is imaginary and Monte Carlo methods fail to converge. This is the reason why on the Lattice one can extract results only up to few terms in the expansion around $\theta=0$, either analytically continuing from imaginary $\theta$ values or computing certain correlators at $\theta=0$. See [1] for an excellent review on the subject.

Some hints on the physics beyond the small $\theta$ regime can be obtained studying the large $N_{c}$ limit [2] where $\theta / N_{c}$ is held fixed. Periodicity under $2 \pi$ shifts and $\theta / N_{c}$ scaling can be reconciled if there are infinite possible vacua, labeled by an integer $k$, which become stable but non-degenerate in the $N_{c}=\infty$ limit. The ground-state energy density, for a given value of $\theta$, is obtained by minimizing,

^e-mail: bigazzi@fi.infn.it 
with respect to $k$, a function of $(\theta+2 \pi k) / N_{c}$. The resulting expression, periodic in $\theta$, shows cusps at particular values of $\theta$, accounting for jumps between different branches.

Remarkably, these features can be explicitly realized in a large $N_{c}$ Yang-Mills model introduced by Witten in [3]. The reason is that the model, under certain assumptions, can be explored at the non-perturbative level and for any value of $\theta$ using the holographic gauge/gravity correspondence [4-6]. The latter is a proposed equivalence map between classes of quantum field theories (QFT) and theories of gravity in at least one extra dimension, which finds its concrete realization in the framework of string theory. Open strings contain gauge degrees of freedom, while closed strings contain gravitons. The open/closed string duality, which naturally emerges in the theory practically implements the gauge/gravity correspondence.

The correspondence is relevant since it works as a duality: the large $N_{c}$, strong 't Hooft coupling regime of, say, a $S U\left(N_{c}\right)$ gauge theory, can be mapped into a classical theory of gravity. Gauge invariant operators correspond to gravity fields, QFT local symmetries are mapped into isometries on the gravity side. Finally, the QFT partition function on a given vacuum, is computed by the on-shell gravity action on the dual background corresponding to that vacuum.

The map between a QFT and a theory of gravity is one-to-one, when the dual pairs are embedded into a consistent ten dimensional string theory model. This feature is crucially based on the properties of the so called $D p$-branes, $p+1$ dimensional hyperplanes over which the open strings can end (here " $D$ " stands for Dirichlet boundary conditions). These $D$-branes are actually physical objects, with their own tension and charge (they are electrically charged under $C_{p+1}$ potentials): as such they deform the ambient spacetime. The resulting curved background and the low energy effective QFT on such $D$-branes are the dual pairs in the game.

When a stack of $N_{c} D 3$-branes in flat space is considered, the low energy QFT is the maximally supersymmetric Yang-Mills theory in $3+1$ dimensions. This is also a conformal field theory (CFT). The dual description is provided by the near-horizon geometry sourced by the D3-branes: the direct product of a five dimensional Anti de Sitter (AdS) spacetime and a five dimensional sphere [4].

In order to describe a non supersymmetric and non conformal Yang-Mills theory one has thus to go beyond this AdS/CFT correspondence. This is the case for the model [3] we will focus on. The starting point is a $5 \mathrm{~d}$ supersymmetric $S U\left(N_{c}\right)$ gauge theory describing the low energy dynamics of $N_{c}$ $D 4$-branes. One of the spatial directions, call it $x_{4}$, is compactified on a circle of radius $1 / M_{K K}$, where antiperiodic boundary conditions on the fermions are imposed. The resulting low energy model is a $4 \mathrm{~d}$ non supersymmetric $S U\left(N_{c}\right)$ Yang Mills coupled with a tower of adjoint massive Kaluza-Klein (KK) modes, with mass scale set by $M_{K K}$. A $\theta$ term can be easily added to the model and the holographic correspondence allows to explore the related physics without encountering any sign problem. To leading order in $\theta / N_{c}$ this has been already done by Witten in [7]. It is not difficult, however, to go beyond this limit and so to consider finite generic values of $\theta$. The corresponding gravity solution has been found in $[8,9]$ and the related physics has been further explored in [10]. The present contribution contains a brief review of the latter work.

We will first provide some details about the gauge theory model and its dual gravity description. Then, we will present a selected class of results, ranging from the $\theta$-dependence of the ground state energy density, the lightest glueball mass and the string tension to that of the finite temperature phase diagram. As we will see, the holographic model qualitatively agrees with available Lattice data in the small $\theta$ regime, confirming that mass scales get reduced by $\theta$ to leading order and providing possible benchmarks for higher order corrections. Moreover, as it is shown in [10], holographic tools allow to extract the $\theta$-dependence of observables like the 't Hooft loop, the baryon mass (vertex) and the entanglement entropy, which are not under control using other techniques. It is also possible to introduce massive quarks and study the physics of the associated QCD-like holographic model, obtaining 
interesting results concerning for example the vacuum structure of the theory or the calculation of the neutron electric dipole moment [11].

\section{The $\theta$ term in Witten's Holographic Yang Mills}

The gauge/gravity dual pair we want to focus on is described by the following actions. On the gravity side, the relevant one is (in string frame) the following consistent truncation of the 10d type IIA supergravity action

$$
S_{\text {gravity }}=\frac{1}{2 k_{0}^{2}} \int d^{10} x \sqrt{-g}\left[e^{-2 \phi}\left(\mathcal{R}+4(\partial \phi)^{2}\right)-\frac{1}{2}\left|F_{4}\right|^{2}-\frac{1}{2}\left|F_{2}\right|^{2}\right] .
$$

Here $2 k_{0}^{2}=(2 \pi)^{7} l_{s}^{8}$ where $l_{s} \equiv \sqrt{\alpha^{\prime}}$ is the string length, $F_{4}=d C_{3}$ is a four-form which is magnetically sourced by the $N_{c} D 4$-branes, $F_{2}=d C_{1}$, and $\phi$ is the dilaton.

The gauge theory action (omitting the contribution of the KK fields) is obtained as the low energy limit of the action for $N_{c} D 4$-branes wrapping a circle $S_{x^{4}}$

$$
S_{\text {gauge }}=\frac{1}{8 \pi g_{s} l_{s} M_{K K}} \int d^{4} x \operatorname{Tr} F^{2}-\frac{i}{32 \pi^{2} l_{s}} \int_{S_{x^{4}}} C_{1} \int d^{4} x \epsilon^{\mu \nu \rho \sigma} \operatorname{Tr} F_{\mu \nu} F_{\rho \sigma} .
$$

Comparing this with (1), one gets the following identifications

$$
\lambda=g^{2} N_{c} \equiv 2 \lambda_{4} \equiv 2 g_{Y M}^{2} N_{c}=4 \pi g_{s} N_{c} l_{s} M_{K K}, \quad \theta+2 \pi k=\frac{1}{l_{s}} \int_{S_{x_{4}}} C_{1},
$$

where $k$ is an integer (accounting for the fact that the integral of $C_{1}$ is gauge invariant only modulo $2 \pi \mathbb{Z}), g_{s}$ is the string coupling and $\lambda_{4}, g_{Y M}$ are introduced to match with a standard notation adopted for Witten's model.

Holography allows to study the gauge theory above by means of a dual classical gravity solution, provided we take the limits $\lambda_{4} \gg 1$ and $N_{c} \gg 1$. Beyond these limits one should add $\alpha^{\prime}$ and string loop corrections to the gravity action.

The family (parameterized by the integer $k$ ) of gravity backgrounds corresponding to the $k$ branches of field theory vacua has been found in $[8,9]$. The metric reads

$$
d s_{10}^{2}=\left(\frac{u}{R}\right)^{3 / 2}\left[\sqrt{H_{0}} d x_{\mu} d x^{\mu}+\frac{f}{\sqrt{H_{0}}} d x_{4}^{2}\right]+\left(\frac{R}{u}\right)^{3 / 2} \sqrt{H_{0}}\left[\frac{d u^{2}}{f}+u^{2} d \Omega_{4}^{2}\right],
$$

where

$$
f=1-\frac{u_{0}^{3}}{u^{3}}, \quad H_{0}=1-\frac{u_{0}^{3}}{u^{3}} \frac{\Theta^{2}}{1+\Theta^{2}} .
$$

The background also includes a running dilaton, a one-form and a four-form field strength given by

$$
e^{\phi}=g_{s}\left(\frac{u}{R}\right)^{3 / 4} H_{0}^{3 / 4}, \quad C_{1}=\frac{\Theta}{g_{s}} \frac{f}{H_{0}} d x^{4}, \quad F_{4}=3 R^{3} \omega_{4},
$$

with $R=\left(\pi g_{s} N_{c}\right)^{1 / 3} l_{s}$ and

$$
\Theta \equiv \frac{\lambda_{4}}{4 \pi^{2}}\left(\frac{\theta+2 k \pi}{N_{c}}\right) .
$$

In the expressions above $\mu=0,1,2,3$ are the $1+3$ Minkowski directions where the Yang-Mills theory is defined, $d \Omega_{4}^{2}$ is the metric of a four-sphere $S^{4}$ of unit radius, $u$ is a radial coordinate $u \in\left[u_{0}, \infty\right)$ - 
holographically mapped to the renormalization group scale of the dual field theory - $x_{4}$ is the compact coordinate of length $2 \pi M_{K K}^{-1}$ and $R$ is a curvature radius. The isometry group of $S^{4}$ corresponds to a global $S O(5)$ symmetry group in the dual field theory; this acts non-trivially on the Kaluza-Klein massive modes signaling that these are not decoupled. Finally $\omega_{4}$ is the volume form of the transverse $S^{4}$, of volume $V_{S^{4}}=8 \pi^{2} / 3$.

The $S_{x_{4}}$ circle shrinks to zero size at $u=u_{0}$ and absence of conical singularities is guaranteed if

$$
u_{0}=\frac{4 R^{3}}{9} M_{K K}^{2} \frac{1}{1+\Theta^{2}},
$$

which implies that the $\left(x_{4}, u\right)$ subspace has the topology of a disk.

The above gravity background is regular and in particular, since $g_{00}\left(u_{0}\right) \neq 0$, it describes a confining gauge theory. This will be made explicit in a moment when the string tension of the model will be computed.

\subsection{The ground-state energy density}

Holography relates the field theory partition function with the (renormalized) on-shell gravity action on the $\theta$-dependent background reviewed above. In particular

$$
Z=e^{-V_{4} f(\theta)} \approx e^{-S_{E \text { on-shell }}^{\text {ren }}}
$$

where $f(\theta)$ is the Yang Mills ground state energy density, $V_{4}$ is the (infinite) 4 d Euclidean spacetime volume and

$$
S_{E \text { on-shell }}^{\text {ren }}=S_{E}+S_{G H}+S_{c . t .} .
$$

Here $S_{E}$ is the Euclidean version of the action (2). Moreover

$$
S_{G H}=-\frac{1}{k_{0}^{2}} \int d^{9} x \sqrt{h} e^{-2 \phi} K,
$$

is the Gibbons-Hawking term, where $h$ is the determinant of the boundary metric and $K$ is the trace of the extrinsic curvature of the boundary. Finally, the counter-term action is given by [12]

$$
S_{c . t .}^{\text {bulk }}=\frac{1}{k_{0}^{2}}\left(\frac{g_{s}^{1 / 3}}{R}\right) \int d^{9} x \sqrt{h} \frac{5}{2} e^{-7 \phi / 3} .
$$

Using the holographic map (10) it follows that

$$
f(\theta)=\min _{k} f(\Theta), \quad \text { where } \quad f(\Theta)=-\frac{2 N_{c}^{2} \lambda_{4}}{3^{7} \pi^{2}} \frac{M_{K K}^{4}}{\left(1+\Theta^{2}\right)^{3}} .
$$

The ground state energy density (see figure 1) turns out to be a periodic function of $\theta$, as expected. To any given interval, of length $2 \pi$, of possible values of $\theta$, it corresponds a value of $k$. For example, $k=0$ when $\theta \in(-\pi, \pi)$. The cusps at $\theta= \pm \pi$ and odd multiples of these values, signal first order $\mathrm{CP}$-breaking phase transitions. In the small $\theta$ limit one gets

$$
f(\theta)-f(0)=\frac{1}{2} \chi_{g} \theta^{2}\left[1+\bar{b}_{2} \frac{\theta^{2}}{N_{c}^{2}}+\bar{b}_{4} \frac{\theta^{4}}{N_{c}^{4}}+O\left(\theta^{6}\right)\right],
$$

with the topological susceptibility given by $\chi_{g}=\lambda_{4}^{3} M_{K K}^{4} /\left(4(3 \pi)^{6}\right)$ and the expansion coefficients given by $\bar{b}_{2}=-\lambda_{4}^{2} /\left(8 \pi^{4}\right), \bar{b}_{4}=5 \lambda_{4}^{4} /\left(384 \pi^{8}\right)$. 


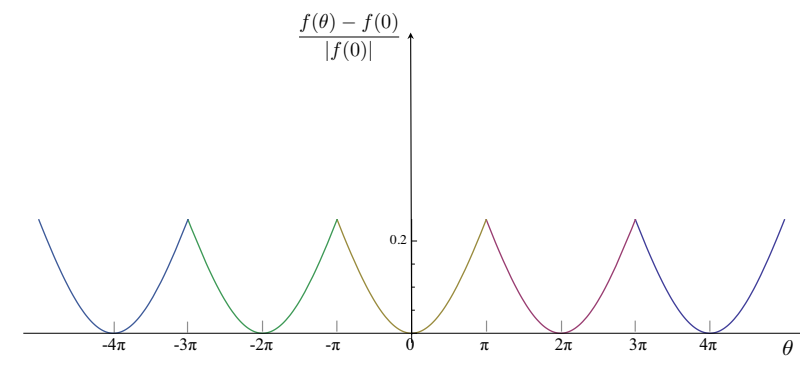

Figure 1. The (normalized) ground state energy density at finite $\theta$. Different colors correspond to different branches of the vacuum energy. The plot has been obtained setting $\lambda_{4} /\left(4 \pi^{2} N_{c}\right)=0.1$.

These results can be compared with those obtained for pure Yang-Mills on the Lattice (see [1]), where extrapolating from the $N_{c}=3,4,6$ results one gets $\bar{b}_{2} \approx-0.2$. The sign of this coefficient matches with that obtained in the holographic model.

Large errors do not allow to fix the sign of $b_{4}$ on the Lattice: recent results $[13,14]$ just provide a bound on its absolute value $\left|b_{4}\right|<0.001$. Our results suggest that $b_{4}$ should have a positive sign.

\subsection{The string tension}

Another standard holographic map [15] relates the VEV of a Wilson loop on a contour $C$ with the (renormalized) Nambu-Goto on-shell action for a fundamental open string whose end-points span that contour

$$
\langle W[C]\rangle \sim e^{-S_{N G}^{r}} .
$$

For a rectangular contour with sides of length $t$ along the time direction and length $L$ along one spatial direction, the Wilson loop allows to obtain the static quark-antiquark potential $V(L)$. In the large $L$ limit in fact $\langle W[C]\rangle \approx e^{-t V(L)}$. Linear confinement of the chromoelectric flux tube implies that $V(L)=T_{s} L$ at large $L$ where $T_{s}$ is the string tension.

The holographic computation of the rectangular Wilson loop just requires minimizing the NambuGoto action of an open string, localized in the transverse $S^{4}$, with worldvolume coordinates $\tau=x^{0} \in$ $[0, t], \sigma=x \in[-L / 2, L / 2]$, and embedded in such a way that $u=u(x)$

$$
S_{N G}=-\frac{1}{2 \pi \alpha^{\prime}} \int d \tau d \sigma \sqrt{-g_{\tau \tau} g_{\sigma \sigma}}=-\frac{1}{2 \pi \alpha^{\prime}} t \int d x \sqrt{-g_{00}\left(g_{x x}+g_{u u} u^{\prime}(x)^{2}\right)},
$$

where $g$ is the (induced) string frame metric. In the large $L$ limit the string profile which minimize this action is well approximated by a bath-tube-shaped configuration: a string stretching for most of its length at $u=u_{0}$, turning vertically, around the extrema $x=-L / 2$ and $x=L / 2$, up to a UV cutoff $u=u_{\Lambda} \rightarrow \infty$ where it is attached to a probe brane. The two vertical parts of the string give divergent contributions to the action when the cutoff is sent to infinity. They are interpreted as due 
to the (infinite) masses of the static quark-antiquark pair and must be subtracted in order to get the renormalized Nambu-Goto action. All in all, in the large $L$ limit one gets

$$
S_{N G}^{r} \approx-\left.\frac{1}{2 \pi \alpha^{\prime}} t \sqrt{-g_{00} g_{x x}}\right|_{u=u_{0}} L,
$$

which in turn, on the $\theta$-deformed background (5), gives the string tension

$$
T_{s}=\frac{2 \lambda_{4}}{27 \pi} M_{K K}^{2} \frac{1}{\left(1+\Theta^{2}\right)^{2}},
$$

which becomes a periodic function after the minimization over $k$ is performed. Around $\theta \rightarrow 0$

$$
T_{s}=\frac{2 \lambda_{4}}{27 \pi} M_{K K}^{2}\left(1-\frac{\lambda_{4}^{2}}{8 \pi^{4}} \frac{\theta^{2}}{N_{c}^{2}}+\frac{3 \lambda_{4}^{4}}{256 \pi^{8}} \frac{\theta^{4}}{N_{c}^{4}}+O\left(\theta^{6}\right)\right) .
$$

Notice that the $O\left(\theta^{2}\right)$ correction is negative. This, together with the expected scaling with $\theta / N_{c}$, is precisely what has been found on the Lattice in [1]

$$
T_{\text {slat }}=T_{\text {slat }}(0)\left[1+\bar{s}_{2} \frac{\theta^{2}}{N_{c}^{2}}+\ldots\right],
$$

where, using the large $N_{c}$ extrapolation of the $N_{c}=3, \ldots, 6$ results one gets $\bar{s}_{2} \approx-0.9$. The holographic result (20) also predicts that the $O\left(\theta^{4}\right)$ correction to the string tension in pure Yang-Mills should be positive.

\subsection{The scalar glueball mass}

Just as in pure Yang-Mills, Witten's model has a mass gap in the glueball spectrum [3]. Each glueball state corresponds to a mode in the dual string picture. The $0^{++}$glueball closely related to that of pure Yang-Mills can be identified with a scalar fluctuation involving the dilaton. The equation of motion for the fluctuation (see [10] for details)

$$
H^{\prime \prime}(u)+\frac{4 u^{3}-u_{0}^{3}}{u\left(u^{3}-u_{0}^{3}\right)} H^{\prime}(u)-\frac{M^{2} R^{3}}{u^{3}-u_{0}^{3}} H(u)=0,
$$

has to be solved by imposing regularity at $u=u_{0}$ and normalizability at $u \rightarrow \infty$. The possible solution exist only if $M^{2}>0$ and the spectrum is discrete. From equation (9) it follows that the mass of the $0^{++}$glueball is formally given by $M(\Theta)=M(\Theta=0)\left(1+\Theta^{2}\right)^{-1 / 2}$. Around $\theta=0$

$$
M(\theta)=M(\theta=0)\left(1-\frac{\lambda_{4}^{2}}{32 \pi^{4}} \frac{\theta^{2}}{N_{c}^{2}}+\frac{3 \lambda_{4}^{4}}{2048 \pi^{8}} \frac{\theta^{4}}{N_{c}^{4}}+O\left(\theta^{6}\right)\right),
$$

where the leading correction in $\theta^{2}$ has a negative sign, as in Lattice Yang-Mills [16]. The holographic model predicts that the $O\left(\theta^{4}\right)$ correction has a positive sign.

\subsection{Finite temperature: $\theta$-dependence of the phase diagram}

Going to finite temperature in Witten's holographic Yang-Mills model, amounts to compactifying the Euclidean time on a circle of length $\beta=1 / T$. There are two possible solutions of the gravity equations 
of motion for which this condition can be satisfied (see e.g. [17]). A first solution is just the Euclidean continuation (with a compact time circle) of the one presented above. This solution corresponds to the confined phase of the gauge theory. The free energy density in this phase has the same expression as in the $T=0$ case (14).

The second solution has a black hole event horizon and corresponds to a deconfined phase. The $S_{x^{4}}$ circle does not shrink anymore and the equations of motion are solved by $C_{1}=\theta d x^{4}$ which implies that $F_{2}=0$. As a result the background metric looses any $\theta$-dependence

$$
\begin{aligned}
& d s^{2}=\left(\frac{u}{R}\right)^{3 / 2}\left[\tilde{f}(u) d x_{0}^{2}+d x_{a} d x^{a}+d x_{4}^{2}\right]+\left(\frac{u}{R}\right)^{-3 / 2}\left[\frac{d u^{2}}{\tilde{f}(u)}+u^{2} d \Omega_{4}^{2}\right], \\
& \tilde{f}(u)=1-\frac{u_{T}^{3}}{u^{3}} .
\end{aligned}
$$

The dilaton and the $F_{4}$ form do not change w.r.t. the $T=0$ solution. In the equation above, $a=1,2,3$. Regularity is now guaranteed by the relation $9 \beta^{2} u_{T}=16 \pi^{2} R^{3}$, where $u_{T}$ is the radial position of the horizon.

The gauge theory free energy density (hence minus the pressure) in the deconfined phase is holographically mapped into that of the black hole background and reads

$$
f_{\text {dec }}=-p_{\text {dec }}=-\frac{1}{6} \frac{256 N_{c}^{2} \pi^{4} \lambda_{4}}{729 M_{K K}^{2}} T^{6},
$$

where the scaling with $T$ follows from the higher dimensional UV completion of the model. The free energy is independent from $\theta$ and thus the topological susceptibility is zero in the deconfined phase. This is actually what one expects in the large $N_{c}$ limit of Yang-Mills, where the susceptibility is exponentially suppressed.

Comparing the free energies (or pressures) in the two phases one gets that the dual field theory experiences a first order confinement-deconfinement phase transition at a critical temperature $T_{c}$ formally given by

$$
T_{c}(\Theta)=\frac{M_{K K}}{2 \pi} \frac{1}{\sqrt{1+\Theta^{2}}},
$$

which corresponds to a periodic function $T_{c}(\theta)$ as shown in figure 2 .

Remarkably the holographic model explicitly realizes the expected but still conjectural (see e.g. [18]) structure of the phase diagram, with triple critical points where the first order deconfinement transition line meets the first order lines related to the CP breaking transitions in the confined phase. One can conjecture that the same cusped structure should appear in pure Yang-Mills at large $N_{c}$.

Around $\theta=0$ we find (at $O\left(\theta^{2}\right)$ the result was already found in [19])

$$
T_{c}(\theta)=\frac{M_{K K}}{2 \pi}\left[1-\frac{\lambda_{4}^{2}}{32 \pi^{4}} \frac{\theta^{2}}{N_{c}^{2}}+\frac{3 \lambda_{4}^{4}}{2048 \pi^{8}} \frac{\theta^{4}}{N_{c}^{4}}+O\left(\theta^{6}\right)\right],
$$

where, again, both the quadratic dependence and the sign of the leading $\theta$-dependent correction, agree with what has been found (for $N_{c}=3$ ) on the Lattice [18]

$$
T_{c}(\theta)_{\text {lat }}=T_{c}(0)_{\text {lat }}\left[1-R_{\theta} \theta^{2}+O\left(\theta^{4}\right)\right], \quad R_{\theta}=0.0175(7) .
$$

Our model, then, predicts that the $O\left(\theta^{4}\right)$ correction has a positive coefficient. It would be nice to check this and the previous predictions on the Lattice.

\section{Acknowledgments}

FB is grateful to the organizers of the Workshop "QCD at Work 2016" for their kind invitation. 


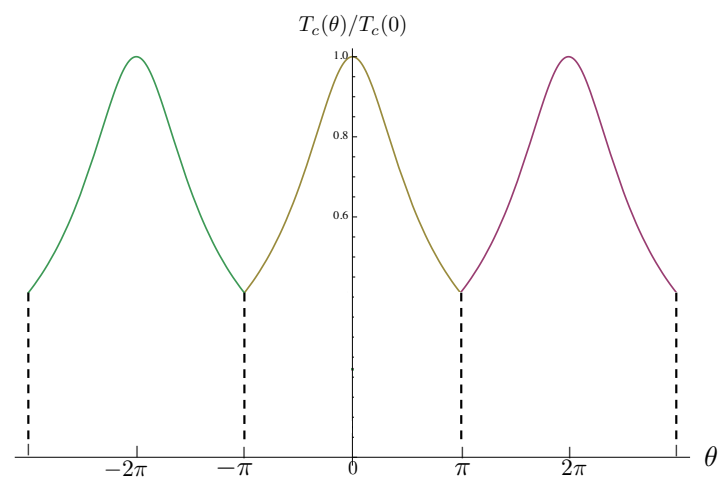

Figure 2. The $(T, \theta)$ phase diagram. Different colors correspond to different branches of the vacuum energy.

\section{References}

[1] E. Vicari and H. Panagopoulos, Phys. Rept. 470, 93 (2009).

[2] E. Witten, Annals Phys. 128, 363 (1980).

[3] E. Witten, Adv. Theor. Math. Phys. 2, 505 (1998).

[4] J. M. Maldacena, Int. J. Theor. Phys. 38, 1113 (1999) [Adv. Theor. Math. Phys. 2, 231 (1998)].

[5] E. Witten, Adv. Theor. Math. Phys. 2, 253 (1998).

[6] S. S. Gubser, I. R. Klebanov and A. M. Polyakov, Phys. Lett. B 428, 105 (1998).

[7] E. Witten, Phys. Rev. Lett. 81, 2862 (1998).

[8] J. L. F. Barbon and A. Pasquinucci, Phys. Lett. B 458, 288 (1999).

[9] S. Dubovsky, A. Lawrence and M. M. Roberts, JHEP 1202, 053 (2012).

[10] F. Bigazzi, A. L. Cotrone and R. Sisca, JHEP 1508, 090 (2015).

[11] L. Bartolini, F. Bigazzi, S. Bolognesi, A. L. Cotrone and A. Manenti, to appear.

[12] D. Mateos, R. C. Myers and R. M. Thomson, JHEP 0705, 067 (2007).

[13] H. Panagopoulos and E. Vicari, JHEP 1111, 119 (2011).

[14] C. Bonati, M. D'Elia, P. Rossi and E. Vicari, " $\theta$ dependence of 4D $S U(N)$ gauge theories in the large- $N$ limit," arXiv:1607.06360 [hep-lat].

[15] J. M. Maldacena, Phys. Rev. Lett. 80, 4859 (1998). S. J. Rey and J. T. Yee, Eur. Phys. J. C 22, 379 (2001).

[16] L. Del Debbio, G. M. Manca, H. Panagopoulos, A. Skouroupathis and E. Vicari, JHEP 0606, 005 (2006).

[17] O. Aharony, J. Sonnenschein and S. Yankielowicz, Annals Phys. 322, 1420 (2007).

[18] M. D’Elia and F. Negro, Phys. Rev. Lett. 109, 072001 (2012); Phys. Rev. D 88, no. 3, 034503 (2013).

[19] F. Bigazzi and A. L. Cotrone, JHEP 1501, 104 (2015). 